\title{
Tracheobronchial malacia and stenosis in children in intensive care: bronchograms help to predict oucome
}

\author{
Robert J Burden, Frank Shann, Warwick Butt, Michael Ditchfield
}

\begin{abstract}
Background-Severe tracheobronchial malacia and stenosis are important causes of morbidity and mortality in children in intensive care, but little is known about how best to diagnose these conditions or determine their prognosis.

Methods-The records of all 62 children in whom one or both of these conditions had been diagnosed by contrast cinetracheobronchography in our intensive care unit in the period 1986-95 were studied.

Results-Seventy four per cent of the 62 children had congenital heart disease; none was a preterm baby with airways disease associated with prolonged ventilation. Fifteen of the children had airway stenosis without malacia; three died because of the stenosis and two died from other causes. Twenty eight of the 47 children with malacia died; only eight children survived without developmental or respiratory handicap. All children needing ventilation for malacia for longer than 14 consecutive days died if their bronchogram showed moderate or severe malacia of either main bronchus (15 cases), or malacia of any severity of both bronchi (three additional cases); all children needing ventilation for malacia for longer than 21 consecutive days died if their bronchogram showed malacia of any severity of the trachea or a main bronchus (three additional cases). These findings were strongly associated with a fatal outcome $(p<0.00005)$; they were present in 21 children (all of whom died) and absent in 26 (of whom seven died, six from non-respiratory causes). They had a positive predictive value for death of $100 \%$, but the lower limit of the $95 \%$ confidence interval was $83.9 \%$ so up to $16 \%$ of patients meeting the criteria might survive.

Conclusion-In this series the findings on contrast cinetracheobronchography combined with the duration of ventilation provided a useful guide to the prognosis of children with tracheobronchomalacia. The information provided by bronchoscopy was less useful.

(Thorax 1999;54:511-517)
\end{abstract}

Keywords: bronchography; tracheobronchomalacia; children; tracheal stenosis

Tracheobronchomalacia is an uncommon condition that causes weakness of the tracheobron- chial tree $e^{1-3}$ and collapse of the airways that is most apparent in expiration. The condition is usually self-limiting, but severe disease can result in prolonged treatment in intensive care and is often fatal.

The tracheobronchial tree can be assessed with many different modalities including noncontrast fluoroscopy, tracheobronchography, computed tomography, magnetic resonance imaging, bronchoscopy, or a combination of all these methods. The diagnosis of tracheobronchomalacia, however, requires a dynamic assessment of the trachea and bronchi throughout a respiratory cycle with demonstration of collapse of the airway in expiration. Bronchoscopy is of limited value because the airway is splinted by the bronchoscope which reduces the amount by which the airways collapse. In addition, small airways or airways beyond a stenosis cannot be examined. Non-contrast fluoroscopy can satisfactorily assess the state of the trachea ${ }^{4}$ but it does not show the bronchi adequately. ${ }^{56}$ Spiral and ultrafast computed tomography ${ }^{6}$ and magnetic resonance imaging with rapid acquisition sequences can show the trachea and main bronchi, but these techniques do not provide optimal information because the airways move in and out of the plane of imaging during a respiratory cycle. ${ }^{7}$ In addition, they are time consuming and often require increased sedation. Tracheobronchography can rapidly and accurately assess the entire airway dynamically without significant morbidity, and it is a simple procedure to perform in children who are already intubated. ${ }^{58}$

Little is known about the natural history of tracheobronchomalacia in children in intensive care, or the severity and location of the airways disease. We have reviewed our experience with tracheobronchial malacia and stenosis in children in our intensive care unit and analysed the findings on cinetracheobronchography.

\section{Methods}

Following local ethical committee approval, we reviewed the records of 62 children admitted to our intensive care unit in whom the diagnosis of either malacia or stenosis of the airways was made following bronchography in the 10 years from January 1986 to December 1995. Chart information was recorded systematically by a clinician (RJB) blinded to the results of bronchographic review. These data included details on associated diagnoses, intubation and ventilation characteristics, all surgical procedures including attempts to correct airway defects, reasons for non-survival, and bronchoscopic findings. 
A telephone survey was conducted (by RJB and WB) to obtain information on the chronic health outcome of surviving children. Parents were questioned about any current symptoms in their children, including the presence of wheeze, cough, stridor, apnoeic or cyanotic episodes, and home oxygen dependence, as well as about the child's exercise tolerance, general development, medications, school absenteeism due to respiratory illness, and the number of lower respiratory tract infections occurring each year. The outcome of the survivors was classified as follows: (1) normal; (2) functionally normal (both intellectually and physically) but requiring medication or medical supervision-for example, a child with chronic asthma on regular medication but able to exercise without restriction and missing less than two weeks of school a year; (3) mild handicap but likely to lead an independent existence-for example, chronic asthma causing restriction of activity, hospital admissions, or loss of more than two weeks of school a year; (4) moderate handicap dependent on care-for example, Down's syndrome; (5) or severe handicap (vegetative, totally dependent on (are). ${ }^{9}$

Bronchography was performed at our hospital in a standardised manner. The patients were lightly anaesthetised, intubated, and were breathing spontaneously. The endotracheal tube was maintained in a high position with the tip in the subglottic region. Opacification of the airways was obtained by bolus injections of small volumes $(1-2 \mathrm{ml})$ of contrast-initially iodised oil (Lipiodol) and more recently iohexol (Omnipaque) $300 \mathrm{mg} / \mathrm{ml}$. The contrast was injected through a small feeding tube passed through a connector in the airway circuit close to the endotracheal tube. Contrast dispersal was obtained by hand ventilating the patient; this enabled opacification of the trachea and first, second, and third generation bronchi. All patients had their tracheobronchograms recorded on video and with radiographic spot films. A pressure monitor was connected to the airway circuit via a Y connector; the study was performed at atmospheric pressure and at positive pressures of up to $20 \mathrm{~cm} \mathrm{H}_{2} \mathrm{O}$.

The bronchograms were retrospectively reviewed by a radiologist (MD) blinded to the clinical data. Up to four affected sites were identified (trachea, left main bronchus, right main bronchus, and peripheral bronchi). Airway malacia was diagnosed when narrowing was present on expiration (mild $=$ diameter $30-50 \%$ of normal, moderate $=10-30 \%$, and severe $=0-10 \%)$ and the diameter increased either on inspiration or with $20 \mathrm{~cm} \mathrm{H}_{2} \mathrm{O}$ of positive end expiratory pressure. A record was kept of the end expiratory pressure required to restore airway patency in airways affected by malacia. Airways were classified as stenosed when the narrowing was fixed and unaffected by positive airway pressure (mild $=$ diameter $60-100 \%$ of normal, moderate $=30-60 \%$, and severe $=0-30 \%)$. Lesions were termed "diffuse" when they involved the entire length of the trachea or bronchus, otherwise they were termed "focal".
Statistical analysis was performed using Fisher's exact test, the Mann-Whitney U test, and exact confidence intervals for proportions (StatXact, Cytel, Cambridge, Massachusetts, USA).

\section{Results}

TRACHEOBRONCHOMALACIA

Forty seven children (30 boys) with tracheobronchomalacia were treated in our intensive care unit during the 10 year period studied. The diagnosis was made before one year of age in 45 of the children $(96 \%)$ and $27(57 \%)$ weighed less than the third centile at the time of diagnosis. Thirteen children (28\%) were born prematurely, of whom 10 had associated complex congenital heart disease. Three children required tracheostomy, of whom one has died and another still has the tracheostomy in place.

Thirty four children $(72 \%)$ had at least one cardiac lesion and $31(66 \%)$ required cardiac surgery. The cardiac lesions present were left to right shunts (ventricular septal defects in 20 children, atrial septal defects in seven, truncus arteriosus in six, patent ductus arteriosus in five, other in one), obstructive right heart lesions (pulmonary atresia in six, absent pulmonary valve in four, tetralogy of Fallot in four, others in three), obstructive left heart lesions (coarctation of the aorta in five, hypoplastic aortic arch in three), and other cardiac lesions (right aortic arch in six, dextrocardia in four, double outlet right ventricle in three, and other in nine). Forty two children $(89 \%)$ had non-cardiac anomalies: dysmorphic syndromes (Di George syndrome in five, cleft lip in three, other in 21), genitourinary (single kidney in three, other in nine), gastrointestinal (reflux in 12, exomphalos in three, oesophageal atresia in three, other in four), respiratory (diaphragmatic palsy in five, vocal cord palsy in three, tracheo-oesophageal fistula in three, other in five), and neurological (epilepsy in four, other in three).

Twenty eight of the 47 children (60\%) died; the median time from diagnosis to death was two months (range 1-9 months) and 23 (82\%) of the 28 children died before 12 months of age. Eighty percent of the children weighed less than the third centile when they died. The commonest cause of mortality was respiratory failure due to airway disease (17/28). Twenty four of the 28 children died in hospital without having returned home since diagnosis.

There were 19 survivors (40\%). All the parents of the surviving children were contacted and participated in the telephone survey. The median survival time from diagnosis was 58 months (range 9-116 months) and the median age at follow up was 64 months (range 12-162 months). Thirty six percent of the survivors were above the 50th centile for weight. Eight children required subsequent admission to hospital due to respiratory illness, and a further eight and 10 children required admission to hospital for cardiac illness or other noncardiorespiratory reasons, respectively. Eight of the 19 survivors (42\%) were normal or 
Table 1 Tracheobronchomalacia: diagnosis, presentation, and outcome in survivors $(n=19)$ ranked by duration of ventilation

\begin{tabular}{|c|c|c|c|c|c|c|c|c|}
\hline \multirow{2}{*}{$\begin{array}{l}\text { Age } \\
\text { (months) } \\
\text { at diagnosis }\end{array}$} & \multirow[b]{2}{*}{ Main diagnosis (or presentation) } & \multirow{2}{*}{$\begin{array}{l}\text { Ventilation } \\
\text { (days) }\end{array}$} & \multicolumn{4}{|c|}{ Bronchogram severity score } & \multirow{2}{*}{$\begin{array}{l}\text { Bronchoscopic } \\
\text { abnormality }\end{array}$} & \multirow[b]{2}{*}{ Handicap } \\
\hline & & & $T$ & $L B$ & $R B$ & $P B$ & & \\
\hline 1 & Pulmonary stenosis & 2 & 1 & 0 & 1 & 0 & None & Moderate \\
\hline 2 & Truncus arteriosis & 3 & 1 & 2 & 1 & 0 & & Normal \\
\hline 0 & Diaphragmatic hernia & 3 & 3 & 0 & 0 & 2 & & Mild \\
\hline 1 & Respiratory failure & 3 & 0 & 1 & 0 & 0 & & Normal \\
\hline 0 & Oesophageal atresia & 3 & 3 & 0 & 0 & 0 & & Functionally normal \\
\hline 3 & Absent pulmonary valve & 4 & 1 & 0 & 0 & 0 & & Mild \\
\hline 0 & Coarctation of the aorta & 6 & 1 & 0 & 0 & 0 & $\mathrm{TM}$ & Normal \\
\hline 2 & Vascular ring & 6 & 0 & 2 & 2 & 0 & & Moderate \\
\hline $1 \overline{1}$ & Coarctation of the aorta & 7 & 1 & 0 & 1 & 0 & & Mild \\
\hline 4 & Oesophageal atresia & 7 & 2 & 2 & 0 & 0 & RBM, LBM & Normal \\
\hline 0 & Absent pulmonary valve & 8 & 0 & 0 & 0 & 2 & & Mild \\
\hline 152 & Pulmonary atresia & 10 & 1 & 0 & 0 & 0 & & Functionally normal \\
\hline 3 & Pulmonary atresia & 11 & 3 & 0 & 0 & 2 & & Severe \\
\hline 11 & Absent pulmonary valve & 12 & 1 & 3 & 3 & 0 & & Moderate \\
\hline 0 & (Respiratory failure) & 14 & 2 & 1 & 0 & 0 & & Mild \\
\hline 0 & Laryngeal cleft & $18^{\star}$ & 2 & 2 & 2 & 0 & None & Mild \\
\hline 7 & Transposition great arteries & 20 & 1 & 0 & 0 & 0 & & Normal \\
\hline 1 & Atrioventricular septal defect & 21 & 0 & 1 & 0 & 0 & & Severe \\
\hline 8 & Pulmonary atresia & 40 & 0 & 0 & 0 & 2 & $\mathrm{RBM}$ & Normal \\
\hline
\end{tabular}

*Ventilated for aspiration rather than tracheobronchomalacia.

$\mathrm{T}=$ trachea; $\mathrm{LB}=$ left main bronchus; $\mathrm{RB}=$ right main bronchus; $\mathrm{PB}=$ peripheral bronchi; $\mathrm{TM}=$ tracheomalacia $\mathrm{LBM}=$ left main bronchomalacia; $\mathrm{RBM}=$ main bronchomalacia; bronchogram severity score $0=$ none, $1=$ mild, $2=$ moderate, $3=$ severe $($ see Methods).

functionally normal, six were mildly handicapped, three were moderately handicapped, and two were severely handicapped (table 1).

The most prevalent respiratory symptom was cough, occurring daily in five children. Twelve children (63\%) required regular medication-four for asthma and five for cardiac disease. In children of school age the mean annual absenteeism due to respiratory disease was eight days (range $0-40$ days). Lower respiratory tract infections occurred between 0 and 10 times a year (median 2). Three children had cyanotic episodes at home but none required home oxygen and none had apnoeic episodes. There was a significant difference between survivors and nonsurvivors in the duration of ventilation $(\mathrm{p}=$ 0.0001 , see tables 1 and 2) and endotracheal intubation $(p=0.0009)$. The geometric mean duration of intubation was 0.96 days $(95 \%$ CI 2.2 to 62.7$)$ in survivors and 27.1 days (95\% CI 4.0 to 182.7) in non-survivors.

The severity and location of malacia airways in our patients are shown in tables 1 and 2 . The trachea was abnormal in 35 of the 47 children $(74 \%)$ and this was an isolated finding in eight $(17 \%)$. Twenty nine $(62 \%)$ and $24(51 \%)$ had lesions affecting the left and right main bronchi, respectively. Nineteen of the 29 lesions affecting the left main bronchus and 19

Table 2 Tracheobronchomalacia: diagnosis, presentation, and cause of death in non-survivors $(n=28)$ ranked by cause of death (respiratory, part respiratory, non-respiratory) and duration of ventilation

\begin{tabular}{|c|c|c|c|c|c|c|c|c|}
\hline \multirow{2}{*}{$\begin{array}{l}\text { Age } \\
\text { (months) } \\
\text { at diagnosis }\end{array}$} & \multirow[b]{2}{*}{ Main diagnosis (or presentation) } & \multirow{2}{*}{$\begin{array}{l}\text { Ventilation } \\
\text { (days) }\end{array}$} & \multicolumn{4}{|c|}{ Bronchogram severity score } & \multirow{2}{*}{$\begin{array}{l}\text { Bronchoscopic } \\
\text { abnormality }\end{array}$} & \multirow[b]{2}{*}{ Cause of death } \\
\hline & & & $T$ & $L B$ & $R B$ & $P B$ & & \\
\hline 3 & Truncus arteriosus & 6 & 3 & 3 & 3 & 0 & & Respiratory \\
\hline 11 & Tetralogy of Fallot & 17 & 2 & 1 & 1 & 0 & TM, LBM & Respiratory \\
\hline 8 & Vascular ring & 19 & 1 & 0 & 2 & 0 & & Respiratory \\
\hline 0 & (Respiratory failure) & 23 & 1 & 2 & 2 & 0 & $\mathrm{TM}$ & Respiratory \\
\hline 11 & Pulmonary atresia & 24 & 2 & 2 & 0 & 2 & & Respiratory \\
\hline 3 & Tetralogy of Fallot & 25 & 0 & 1 & 3 & 2 & & Respiratory \\
\hline 1 & $\begin{array}{l}\text { Anomalous pulmonary venous } \\
\text { drainage }\end{array}$ & 28 & 0 & 2 & 3 & 0 & & Respiratory \\
\hline 1 & Vascular ring & 29 & 0 & 1 & 1 & 1 & None & Respiratory \\
\hline 3 & Absent pulmonary valve & 35 & 1 & 1 & 1 & 0 & & Respiratory \\
\hline 5 & Vascular ring & 37 & 2 & 0 & 2 & 0 & TM, RBM & Respiratory \\
\hline 2 & Absent pulmonary valve & 56 & 2 & 0 & 2 & 0 & & Respiratory \\
\hline 0 & (Respiratory failure) & 60 & 1 & 1 & 0 & 0 & & Respiratory \\
\hline 5 & Cystic right lung & 65 & 1 & 0 & 2 & 0 & & Respiratory \\
\hline 4 & Pulmonary atresia & 68 & 2 & 2 & 2 & 2 & LBM, PBM & Respiratory \\
\hline 0 & Pulmonary stenosis & 72 & 3 & 0 & 0 & 0 & $\mathrm{TM}$ & Respiratory \\
\hline 13 & Hypoplastic aortic arch & 85 & 2 & 2 & 2 & 0 & Hypoplastic LB & Respiratory \\
\hline 0 & Truncus arteriosus & 85 & 2 & 3 & 3 & 0 & & Respiratory \\
\hline 11 & Atrioventricular septal defect & 6 & 0 & 2 & 0 & 2 & & Cardiorespiratory \\
\hline 0 & Pulmonary atresia & 15 & 0 & 3 & 3 & 0 & & Cardiorespiratory \\
\hline 0 & Interrupted aortic arch & 20 & 1 & 2 & 1 & 0 & & Cardiorespiratory \\
\hline 12 & (Respiratory failure) & 2 & 1 & 0 & 0 & 0 & $\mathrm{TM}$ & Neurological \\
\hline 3 & Vascular ring & 10 & 1 & 1 & 0 & 0 & & Neurological \\
\hline 9 & Treacher Collins syndrome & 10 & 0 & 2 & 0 & 0 & LBM & Neurological \\
\hline 7 & Transposition of great arteries & 11 & 0 & 2 & 0 & 0 & TM, LBM, RBM & Multisystem \\
\hline 0 & Coarctation aorta & 19 & 2 & 0 & 0 & 0 & None & Gastrointestinal \\
\hline 2 & Pulmonary atresia & 19 & 3 & 3 & 3 & 0 & & Gastrointestinal \\
\hline 1 & VSD, ASD & 40 & 2 & 1 & 0 & 0 & & Multisystem \\
\hline 0 & Hypoplastic aortic arch & 116 & 3 & 3 & 3 & 3 & LBM & Haemorrhage \\
\hline
\end{tabular}

$\mathrm{T}=$ trachea $\mathrm{LB}=$ left main bronchus $; \mathrm{RB}=$ right main bronchus $; \mathrm{PB}=$ peripheral bronchi $\mathrm{TM}=$ tracheomalacia $; \mathrm{LBM}=$ left main bronchomalacia; $\mathrm{RBM}=$ right main bronchomalacia; $\mathrm{PBM}=$ peripheral bronchomalacia; bronchogram severity score $0=$ none, 1 $=$ mild, 2 = moderate, 3 = severe (see Methods). 
Table 3 Airway stenosis: diagnosis, presentation, and outcome $(n=15)$

\begin{tabular}{|c|c|c|c|c|c|c|c|c|c|}
\hline \multirow{2}{*}{$\begin{array}{l}\text { Age (months) } \\
\text { at diagnosis }\end{array}$} & \multirow{2}{*}{$\begin{array}{l}\text { Main diagnosis } \\
\text { (or presentation) }\end{array}$} & \multirow{2}{*}{$\begin{array}{l}\text { Ventilation } \\
\text { (days) }\end{array}$} & \multicolumn{4}{|c|}{$\begin{array}{l}\text { Bronchogram } \\
\text { severity score }\end{array}$} & \multirow{2}{*}{$\begin{array}{l}\text { Bronchoscopic } \\
\text { diagnosis }\end{array}$} & \multirow[b]{2}{*}{ Handicap } & \multirow[b]{2}{*}{ Cause of death } \\
\hline & & & $T$ & $L B$ & $R B$ & $P B$ & & & \\
\hline 2 & (Stridor) & 0 & 2 & 0 & 0 & 0 & TS & Normal & \\
\hline 8 & (Stridor) & 0 & 3 & 2 & 0 & 0 & TS & Normal & \\
\hline 5 & Vascular ring & 4 & 1 & 0 & 0 & 0 & & Severe & \\
\hline 2 & (Respiratory failure) & 5 & 2 & 0 & 0 & 0 & TS & Mild & \\
\hline 49 & Vascular ring & 6 & 1 & 0 & 0 & 0 & TS & Normal & \\
\hline 4 & Atrioventricular septal defect & 8 & 3 & 0 & 0 & 0 & & Moderate & \\
\hline 2 & Vascular ring & 9 & 3 & 0 & 0 & 0 & & Normal & \\
\hline 2 & Vascular ring & 10 & 2 & 0 & 0 & 0 & & Mild & \\
\hline 0 & Interrupted aortic arch & 23 & 0 & 2 & 2 & 0 & & $\begin{array}{l}\text { Functionally } \\
\text { normal }\end{array}$ & \\
\hline 0 & Coarctation aorta & 55 & 1 & 1 & 1 & 0 & & $\begin{array}{l}\text { Functionally } \\
\text { normal }\end{array}$ & \\
\hline 15 & Patent ductus & 1 & 2 & 0 & 0 & 0 & TS & Died & Respiratory \\
\hline 0 & Vascular ring & 7 & 3 & 0 & 0 & 0 & TS & Died & Multiorgan \\
\hline 5 & Vascular ring & 13 & 2 & 0 & 0 & 0 & TS & Died & Respiratory \\
\hline 3 & Patent ductus & 20 & 1 & 0 & 0 & 0 & TS & Died & Cardiorespiratory \\
\hline 2 & Interrupted aortic arch & 60 & 0 & 3 & 0 & 0 & & Died & Respiratory \\
\hline
\end{tabular}

$\mathrm{T}=$ trachea $\mathrm{LB}=$ left main bronchial stenosis; $\mathrm{RB}=$ right main bronchial stenosis; $\mathrm{PB}=$ peripheral bronchi; $\mathrm{TS}=$ tracheal stenosis; bronchogram severity score: $0=$ none, $1=$ mild, $2=$ moderate, $3=$ severe (see Methods).

of the 24 lesions affecting the right main bronchus occurred in association with tracheomalacia. Ten children $(21 \%)$ had more distal lesions. Thirty nine percent of tracheal lesions were focal compared with $90 \%$ of lesions in the main and peripheral bronchi. Thirteen children had evidence of airway stenosis in addition to malacia; these affected the same airway in eight children and separate airways in five.

All children who needed ventilation for malacia for longer than 14 days died if they had moderate or severe malacia of either main bronchus (15 cases), or malacia of any severity of both main bronchi (three additional cases); all children who needed ventilation for malacia for longer than 21 days died if they had malacia of any severity of the trachea or a main bronchus (three additional cases). None of the children who survived had these findings. These criteria were present in 21 children (all of whom died) and absent in 26 (of whom seven died), giving a sensitivity of $75.0 \%$ (exact $95 \%$ CI 55.1 to 89.3 ), specificity of $100 \%$ (95\% CI 82.3 to 100 ), positive predictive value of $100 \%$ (95\% CI 83.9 to 100$)$, negative predictive value of $73.1 \%$ (95\% CI 52.2 to 88.4 ), and a $p$ value of $<0.00005$ (Fisher's exact test). If the 11 children who died from non-respiratory causes are excluded (the criteria were present in five), the sensitivity is $94.1 \%$ (exact $95 \%$ CI 71.3 to 99.9 ), specificity $100 \%$ (82.3 to 100 ), positive predictive value $100 \%$ (79.4 to 100$)$, and negative predictive value $95.0 \%$ (75.1 to 99.9$)$.

The amount of positive end expiratory pressure required at bronchography to completely expand affected airways ranged between 10 and $20 \mathrm{~cm} \mathrm{H}_{2} \mathrm{O}$; there was no relationship between the amount of positive end expiratory pressure required and either survival or the duration of continuous positive airways pressure, intubation, or ventilation. No child had a severe exacerbation of respiratory failure precipitated by the bronchogram, perhaps because great care was taken to restrict the amount of contrast medium used, particularly when iodised oil was being instilled.
For the admission during which tracheobronchomalacia was diagnosed the median duration of ventilation was 7 (range 2-40) days for survivors and 19 (2-116) days for nonsurvivors $(\mathrm{p}=0.0001)$. The median time in intensive care was 22 (range 3-108) days for survivors and 31 (5-144) days for nonsurvivors. The median time in hospital was 80 (range 14-270) days for survivors and 62 (2-250) days for non-survivors.

STENOSIS

Fifteen children (eight boys) in intensive care were diagnosed with airway stenosis by tracheobronchography (table 3). In 11 cases the diagnosis was made before the age of six months, with eight children weighing less than the third centile. Twelve children had associated cardiac lesions (five had vascular rings) and 14 children had non-cardiac anomalies. Five children were born prematurely. Five children died; the median time from diagnosis to death was 8.3 months (range 1.7-23) and the median age at death was 12.5 months. The parents of all 10 survivors contributed to the telephone survey; six of the 10 children were normal or functionally normal. The commonest symptom was cough which occurred on most days in seven children. Stridor was present every day in only one survivor.

Tracheobronchography demonstrated that tracheal stenosis occurred in 13 patients and that it was an isolated finding in 11 cases. In four cases stenosis occurred in the main bronchi. There were no stenoses seen more peripherally. Thirty three percent of lesions in the trachea and all lesions seen affecting the main bronchi were focal. There was no relationship between the number, site, or severity of stenotic lesions seen on the bronchogram and survival.

\section{SURGERY}

Thirteen of the 62 patients underwent attempts at definitive surgical repair of a major airway (table 4), usually because of failure to wean them from mechanical ventilation. Eleven operations were performed on the 
Table 4 Main lesion and outcome in 13 children undergoing surgery

\begin{tabular}{llll}
\hline Main lesion & Focalldiffuse & Surgery performed & Outcome \\
\hline Main bronchial stenosis & Focal & Resection and end to end anastomosis & Alive \\
Peripheral bronchomalacia & Focal & Bronchoplasty & Alive \\
Tracheal stenosis and malacia & Focal & Tracheopexy & Alive \\
Tracheomalacia & Focal & Tracheopexy & Alive \\
Tracheal stenosis & Focal & Resection and end to end anastomosis & Alive \\
Tracheal stenosis & Focal & Resection and end to end anastomosis & Dead \\
Tracheomalacia & Diffuse & Aortopexy & Dead \\
Tracheomalacia & Diffuse & Pericardial patch repair & Dead \\
Tracheobronchomalacia & Diffuse & Aortopexy & Dead \\
Tracheal stenosis & Diffuse & Tracheoplasty & Dead \\
Tracheal stenosis & Diffuse & Pericardial patch repair & Dead \\
Tracheal stenosis and malacia & Diffuse & Arteriopexy & Alive \\
Tracheal stenosis & Diffuse & Pericardial patch repair & Alive \\
\hline
\end{tabular}

trachea and two on the main bronchi. Six had focal lesions demonstrated by tracheobronchography and five survived after surgery to the affected airway. Seven had diffuse disease and only two survived.

\section{BRONCHOSCOPY}

Twenty five of the 62 patients were investigated by bronchoscopy as well as bronchography near the time of the original diagnosis of either airway stenosis or malacia (tables 1-3). There was agreement about the diagnosis in 12 of the 25 cases and in 10 of the 12 the abnormalities were isolated to the trachea. There was disagreement about the diagnosis in 13 of the 25 cases and in 12 of these the discrepancy involved lesions beyond the trachea; six of these cases had airway abnormalities in the major bronchi or periphery that were detected by bronchography but not by bronchoscopy. A fibreoptic $1.8 \mathrm{~mm}$ bronchoscope was available and was usually used in small infants.

\section{Discussion}

Tracheobronchomalacia was first described in 1952 in three infants ${ }^{1}$ and, since then, many classifications have been proposed. Primary malacia results from immature tracheobronchial cartilages occurring in isolation or in association with congenital conditions such as heart disease, oesophageal atresia, and tracheooesophageal fistula. Secondary malacia results from degeneration of previously normal cartilage and is associated with extrinsic vascular compression, bronchial neoplasms, recurrent bronchitis, gastro-oesophageal reflux, long term intubation, and tracheostomy. ${ }^{23}$ Tracheobronchomalacia has been classified according to the histopathological and bronchoscopic changes seen in the condition ${ }^{10}$ and also according to the degree of luminal narrowing seen during coughing. ${ }^{11}$

The severity of the symptoms depends on the location, length, and severity of the abnormal airway segments. ${ }^{10}$ In children, symptoms include stridor, wheeze, cough, hyperextension of the neck, recurrent respiratory tract infections, cyanotic spells (especially when crying or passing stool), and reflex apnoea. In adults, chronic cough, wheeze, and sputum production predominate. The symptoms associated with mild cases of tracheobronchomalacia generally resolve spontaneously. ${ }^{11}$ Some patients with severe disease respond favourably to conservative measures including oxygen, endotra- cheal suctioning, postural drainage, and positive end expiratory pressure through an endotracheal tube or tracheostomy. Bronchodilators and steroids may improve peripheral obstructive airways disease and hence reduce the dynamic compression of the large airways. $^{3}$ Surgery to the airway is usually reserved for patients in whom medical treatment has failed and is generally performed after underlying surgical causes such as congenital heart disease have been corrected. Procedures include anterior tracheopexy, tracheal stenting, airway implants, and resection of disease segments; they are reported to be more successful when disease is focal rather than diffuse, ${ }^{3} 101213$ although there are no controlled trials of these procedures.

We reviewed all children with tracheobronchomalacia who had received treatment in our intensive care unit over a 10 year period. Contrast cinetracheobronchography was found to be highly predictive of death or survival. All children who needed ventilation for malacia for longer than 14 days died if they had moderate or severe malacia of either main bronchus, or malacia of any severity of both main bronchi (table 2); all children who needed ventilation for malacia for longer than 21 days died if they had malacia of any severity of the trachea or a main bronchus. None of the children who survived had these findings, although one child with moderate malacia of both main bronchi needed ventilation for 18 days because he had a very large laryngeal cleft. The findings were present in 21 children, all of whom died, and absent in 26 of whom seven died, six from non-respiratory causes. Although these criteria had $100 \%$ specificity and positive predictive value for death in our series $(p<0.00005)$, the lower limit of the $95 \%$ confidence interval was $82.3 \%$ for the specificity and $83.9 \%$ for the positive predictive value, so that up to $16 \%$ of patients fulfilling these criteria might survive. The clinicians were aware of the bronchography results at the time that these patients died, but this is very unlikely to have led to a decision to discontinue mechanical ventilation because the prognostic implications of bronchograms were not known then. Indeed, the main reason for doing this study was to determine whether the bronchogram provides information about the prognosis of bronchomalacia.

We found that children with severe tracheobronchomalacia who needed prolonged venti- 
lation for malacia had a very high mortality rate, and malacia of the main bronchi carried a worse prognosis than tracheomalacia. Although these findings provide a very useful guide to the prognosis, they should not be used in isolation as an indication to withdraw treatment; this was a retrospective study, there were only 28 deaths (six from non-respiratory causes), and the clinicians were aware of the bronchogram results.

We found that bronchoscopy was much less sensitive than bronchography in detecting lesions affecting the main bronchi, and the presence of malacia of the main bronchi was the main factor that determined whether a child died or survived. Our findings suggest that contrast cinetracheobronchography is more useful than bronchography for the diagnosis of tracheobronchomalacia, and this has been the experience of other investigators. ${ }^{81415}$ When bronchography is performed the endotracheal tube must be high in the trachea and it is very important that the patient has active spontaneous respiration (bronchomalacia may be missed if the patient is heavily sedated). The complication rate is very low if very small amounts of a non-ionic contrast medium are used.

Tracheobronchomalacia is a rare and serious condition associated with a high mortality in children in intensive care, and it is an important cause of prolonged intubation and ventilation. The median duration of intubation in our population was 12 days for survivors and 30 days for non-survivors, and for ventilation was seven days for survivors and 19 days for non-survivors. The median duration of stay in our intensive care unit for all patients is one day (reflecting the short length of stay in paediatric intensive care in Victoria ${ }^{16}$ ). Death from malacia is commonly the result of respiratory failure and an inability to wean from ventilation; children who die usually succumb within a few months of the original diagnosis of malacia.

Less than half the surviving children in our study were normal or functionally normal at follow up, with only eight of the 19 survivors having both normal development and exercise tolerance. However, cyanotic and apnoeic episodes, which are life-threatening accompaniments of malacia, rarely occurred, and the overall respiratory health of the survivors was generally good (table 1 ).

Thirteen of the 47 patients with malacia were born prematurely and eight of them died. None had developed bronchopulmonary dysplasia; intermittent positive pressure ventilation was indicated for reasons other than hyaline membrane disease and the diagnosis of malacia was made early in their stay in intensive care (after a median of seven days). Prolonged ventilation of premature infants with hyaline membrane disease is associated with bronchopulmonary dysplasia and a tendency towards increased airway collapsibility (acquired tracheobronchomalacia). ${ }^{3}$ These patients may survive after many months of intermittent positive pressure ventilation or continuous positive airways pressure. ${ }^{17}$ The findings on contrast cinebronchography have different implications in these patients because chronic respiratory failure may be due to bronchopulmonary dysplasia as well as bronchomalacia.

Tracheal and bronchial stenosis are also rare conditions. ${ }^{18}$ We reviewed 15 patients in whom cinetracheobronchography had shown airway stenosis without malacia while they were patients in our intensive care unit. Thirteen children had tracheal lesions and two of these had additional bronchial lesions. The remaining two children had stenosis confined to the major bronchi. This suggests that stenotic lesions are commonly confined to the trachea, in contrast to malacia where lesions are often widespread in the tracheobronchial tree. There was a high incidence of associated anomalies, particularly vascular rings and prematurity. Malacia and stenosis coexisted in 13 patients, indicating that neither represents a separate clinical entity in critically ill infants and children.

Thirteen children with malacia or stenosis of the airways underwent attempts at surgical repair, mainly after failure to wean from mechanical ventilation. Surgical repair of focal lesions had a better outcome than repair of diffuse lesions. Contrast cinebronchography provides a record of the length of affected segments and assists in the planning for surgical repair.

Tracheobronchomalacia is rare, but children with this condition who are in intensive care often need prolonged respiratory support and have a high mortality rate. In contrast to bronchoscopy, cinetracheobronchography provides detailed information about the lesions in tracheobronchial malacia as well as stenosis, and it provides information that helps predict which children are likely to survive. Because many centres do not perform tracheobronchograms in high risk patients and other investigations have a much lower sensitivity, tracheobronchomalacia is probably much more common in ventilator dependent children than is generally realised.

We acknowledge the help of Sue Stubbings in Medical Records at the Royal Children's Hospital, and Bernard Higgins at the Department of Mathematics, Portsmouth University. There was no financial support for this work.

1 Hollinger PH, Johnston KC, Parchet VN, et al. Congenital malformations of the trachea, bronchi, and lung. Ann Otol Rhinol Laryngol 1952;61:1104-59.

2 Johnson TH, Mikita JJ, Wilson RJ, et al. Acquired Johnson TH, Mikita JJ, Wilson RJ, et

3 Sotomayor JL, Godinez RI, Borden S, et al. Large airway Sotomayor JL, Godinez RI, Borden S, et al. Large airway
collapse due to acquired tracheobronchomalacia in infancy. Am f Dis Child 1986;140:367-71.

4 Joseph PM, Berdon WE, Baber DH, et al. Upper airway obstruction in infants and small children. Radiology 1976;121:143-8.

5 Riebello T, Wartner R. Use of non-ionic contrast media for tracheobronchography in neonates and young infants. Eur f Radiol 1990;11:120-4.

6 Shepard JO, Grillo HC, Bhalla M, et al. Inspiratoryexpiratory chest $\mathrm{CT}$ in evaluation of large airway disease. Presented at the Radiological Society North America Meeting, Chicago, Illinois. Radiology 1994;193(P):181

7 Brody S, Kuhn JP, Siedel FG, et al. Airway evaluation in children with use of ultrafast CT: pitfalls and recommendations. Radiology 1993;178:181-4.

8 MacIntyre P, Peacock C, Gordon I, et al. Use of tracheobronchography as a diagnostic tool in ventilatordependent infants. Crit Care Med 1998;26:755-9.

9 Butt W, Shann F, Tibballs J, et al. Long-term outcome of Butt W, Shann F, Tibballs J, et al. Long-term outcome of
children after intensive care. Crit Care Med 1990;18:961-5. 
10 Benjamin B. Tracheomalacia in infants and children. Ann Otol Rhinol Laryngol 1984;93:438-42.

11 Cogbill TH, Moore FA, Accurso FJ, et al Primary tracheomalacia. Ann Thorac Surg 1983;35:538-41.

12 Cohen D Tracheopexy: aorto-tracheal suspension for severe tracheomalacia. Aust Paediatr $\mathcal{F}$ 1981;17:117-21.

13 Mair EA, Parsons DS, Lally KP. Treatment of severe bronchomalacia with expanding endobronchial stents. Arch Otolaryngol Head Neck Surg 1990;116:1087-90.

14 Levy M, Glick B, Springer C, et al. Bronchoscopy in children: experience with 110 investigations. Am f Dis Child 1983;137:14-6.
15 Bramson RT, Sherman JM, Blickman JG. Paediatric bronchography performed through the flexible bronchobronchography performed through
scope. Eur f Radiol 1993;16:158-61.

16 Pearson G, Shann F, Barry P, et al. Should paediatric intensive care be centralised? Trent versus Victoria. Lancet 1997;349:1213-7.

17 Wiseman NE, Duncan PG, Cameron CB. Management of tracheobronchomalacia with continuous positive airway pressure. F Pediatr Surg 1985;20:489-93.

18 Hoffer ME, Tom LW, Wetmore RF, et al. Congenital tracheal stenosis. Arch Otolaryngol Head Neck Surg 1994;120:449-53. 